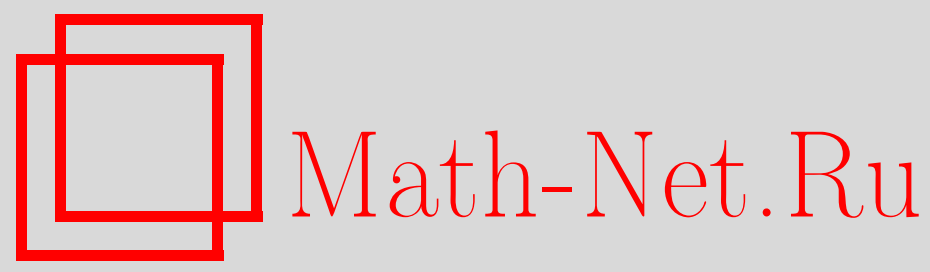

Д. Реповш, П. В. Семенов, О связи между невыпуклостью множества и невыпуклостью его $\varepsilon$-окрестностей, Матем. заметки, 2001, том 70, выпуск 2, 246-259

DOI: https://doi.org/10.4213/mzm738

Использование Общероссийского математического портала Math-Net.Ru подразумевает, что вы прочитали и согласны с пользовательским соглашением http://www . mathnet.ru/rus/agreement

Параметры загрузки:

IP : 54.197 .130 .99

26 апреля 2023 г., 13:41:56

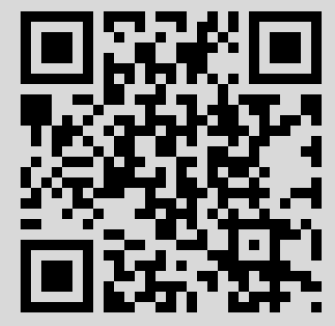




\title{
О СВЯЗИ МЕЖДУ НЕВЫПУКЛОСТЬЮ МНОЖЕСТВА И НЕВЫПУКЛОСТЬЮ ЕГО $\varepsilon$-ОКРЕСТНОСТЕЙ
}

\author{
Д. Реповш, П.В. Семёнов
}

\begin{abstract}
С каждым замкнутым подмножеством $P$ банахова пространства ассоциируется некоторая числовая функция $\alpha_{P}$, характеризующая невыпуклость этого множества. Неравенства типа $\alpha_{P}(\cdot)<1$ обеспечивают хорошие топологические свойства множества $P$ : стягиваемость, экстензорность и т.п. В заметке построены примеры множеств, у которых функции невыпуклости сколь угодно тесных окрестностей существенно отличаются от функции невыпуклости самого множества. С другой стороны, доказано, что в равномерно выпуклых банаховых пространствах условия типа "функция невыпуклости меньше единицы" устойчивы относительно взятия $\varepsilon$-окрестностей множеств.

Библиография: 17 названий.
\end{abstract}

1. Введение. Пусть $P$ - непустое замкнутое подмножество нормированного пространства $B$, и пусть открытый шар $D$ радиуса $r$ пересекает $P$. Число

$$
\delta(P, D)=\sup \left\{\frac{\operatorname{dist}(q, P)}{r} \mid q \in \operatorname{conv}(P \cap D)\right\}
$$

естественно оценивает относительную невьпуклость пересечения множества $P$ с шаpom $D$.

ОПРЕДЕЛЕНИЕ 1.1. (а) Функиия невыпуклости $\alpha_{P}(\cdot)$ ставит в соответствие каждому числу $r>0$ супремум множества всех чисел $\delta(P, D)$ по всем открытым шарам $D$ радиуса $r$.

(б) Множество $P$ назовем $\alpha$-nаравыпуклым, если его функция невыпуклости поточечно на $(0, \infty)$ не превосходит функции $\alpha$.

(в) Множество $P$ назовем $\alpha$-паравыпукльмм $c$ фиксированной точностью $\delta>0$, если его функция невьпуклости поточечно на $(\delta, \infty)$ не превосходит функции $\alpha$.

В случае постоянства функции $\alpha$ определение 1.1(б) совпадает с определением паравыпуклости, предложенным Майклом в [1]. Ясно, что 0-паравьпуклость эквивалентна выпуклости. Функциональная паравьпуклость была рассмотрена в [2], а функции невыпуклости явно были введены в [3]. Возможны различные варианты в определении 1.1(a),

Работа первого автора выполнена при поддержке Министерства науки и техники Республики Словения, грант № J1-0885-0101-98, работа второго автора выполнена при поддержке Российского фонда фундаментальных исследований, гранты № 99-01-00009 и № 99-01-00235. 
полезные в той или иной конкретной ситуации. Если вместо открытых шаров рассматривать замкнутые, то получится определение замкнутой функции невыпуклости. Если рассматривать не все точки $q \in \operatorname{conv}(P \cap D)$, а только чебышевские центры (чебышевский центр в случае равномерной вьпуклости нормы в $B$ ) выпуклого ограниченного множества $\operatorname{conv}(P \cap D)$, то получится определение иентральной функиии невыпуклости. Или же, для каждого конечного подмножества $K \subset P$ чебышевского радиуса $r$ естественно рассмотреть число

$$
\delta_{*}(P, K)=\sup \left\{\frac{\operatorname{dist}(q, P)}{r} \mid q \in \operatorname{conv} K\right\}
$$

и определить внутреннюю функиию невыпуклости множества $P$, которая числу $r$ ставит в соответствие супремум множества всех чисел $\delta_{*}(P, K)$ по всем конечным $K \subset P$ чебьшевского радиуса $r$. Между различными функциями невьпуклости имеются определенные соотношения: если $\alpha_{P}^{\text {in }}$ - внутренняя функция невьпуклости множества $P$, a $\alpha_{P}^{\mathrm{cl}}-$ его замкнутая функция невьпуклости, то для всех $r>0$ выполнены неравенства

$$
\alpha_{P}(r) \leqslant \alpha_{P}^{\mathrm{in}}(r) \leqslant \alpha_{P}^{\mathrm{cl}}(r)=\alpha_{P}(r+0) .
$$

В частности, функции невыпуклости имеют пределы справа (и, на самом деле, непрерьвны слева).

Контроль за невьпуклостью множества на самом деле состоит в контроле за некоторьм итеративным поведением функции невыпуклости этого множества. Для последнего с каждой функцией $\alpha:(0, \infty) \rightarrow[0, \infty)$ ассоциируем "геометрическую со знаменателем $\alpha "$ прогрессию:

$$
q_{\alpha}^{0}(t)=t, \quad q_{\alpha}^{1}(t)=\alpha(t) \cdot t, \quad q_{\alpha}^{n+1}(t)=\alpha\left(q_{\alpha}^{n}(t)\right) \cdot q_{\alpha}^{n}(t)
$$

При этом функция $\alpha$ доопределяется в нуле: $\alpha(0)=0$.

ОПРЕДЕЛЕНИЕ 1.2. (а) Функция $\alpha:(0, \infty) \rightarrow[0, \infty)$ называется геометрически суммируемой, если функциональный ряд $\sum_{n=0}^{\infty} q_{\alpha}^{n}(\cdot)$ поточечно всюду сходится.

(б) Функция $\alpha$ меньше единииы слева, если она имеет строгую геометрически суммируемую мажоранту; обозначение: $\alpha<1_{-0}$.

Нетрудно видеть [2], что если все верхние правые пределы функции $\alpha:(0, \infty) \rightarrow[0, \infty)$ меньше единицы на полуоси $[0, \infty)$, то $\alpha<1_{-0}$.

Проблема контролируемого отказа от выпуклости в селекционных теоремах для многозначных отображений допускает следующее решение (стандартные сведения о теории многозначных отображений см. [4]-[8]).

ТеОрема 1.3 [3], [9]. Пусть $F: X \rightarrow 2^{Y}$ - полунепрерывное снизу замкнутозначное отображение паракомпакта $X$ в банахово пространство $Y$, и пусть

$$
\sup \left\{\alpha_{F(x)} \mid x \in X\right\}<1_{-0}
$$


Тогда F имеет непрерывную однозначную селекиию.

Поэтому если функция невьпуклости некоторого множества меньше единицы слева, то с топологической точки зрения это множество устроено идеально: оно есть абсолютный экстензор (и локальньй абсолютньй экстензор) для класса паракомпактных пространств. В частности, оно стягиваемо и локально стягиваемо.

В настоящей работе исследуется устойчивость такого рода свойств множеств относительно взятия их метрических окрестностей. Более конкретно вопрос звучит так. Пусть функция невьпуклости некоторого множества $P$ меньше единицы слева. Верно ли тогда, что и функции невьпуклости $\varepsilon$-окрестностей этого множества также меньше единицы слева?

В работе построен пример (см. теорему 2.1), показьвающий, что ответ на такой вопрос, вообще говоря, отрищателен: уже в четырехмерном банаховом пространстве оказьвается возможной ситуация, когда паравьпуклое множество имеет сколь угодно тесные не паравыпуклые $\varepsilon$-окрестности. С другой стороны (см. теорему 2.3), в банаховых пространствах с достаточно гладкой единичной сферой ответ все же положителен. С третьей стороны (см. предложение 2.4), даже в евклидовой плоскости функции невыпуклости $\varepsilon$-окрестностей некоторой кривой могут не сходиться к функции невьпуклости самого множества при $\varepsilon \rightarrow 0$, т.е. невыпуклость сколь угодно тесных окрестностей такой кривой может существенно отличаться от невьпуклости самого множества (хотя и быть при этом строго меньше единицы).

\section{2. Формулировки результатов.}

ТЕОрема 2.1. В четырехмерном банаховом пространстве $Y=l_{\infty}^{3} \oplus_{2} \mathbb{R}$ снормой

$$
\|(x, y, z, t)\|=\sqrt{(\max \{|x|,|y|,|z|\})^{2}+t^{2}}
$$

существует одномерное q-паравыпуклое подмножество $P, 0 \leqslant q<1$, и существует последовательность положительных чисел $\varepsilon_{n} \rightarrow 0$ такая, что у замкнутых $\varepsilon_{n}$-окрестностей $\bar{D}\left(P, \varepsilon_{n}\right)=P_{n}$ множества $P$ функиии невыпуклости $\alpha_{P_{n}}$ тождественно равны единице на некоторых интервалах $\left(0, \tau_{n}\right) \subset(0, \infty)$.

Подчеркнем, что равенство единище есть максимально “плохой" возможный случай для функций невьпуклости. Доказательство теоремы 2.1, грубо говоря, основано на том факте, что в банаховом пространстве $Y=l_{\infty}^{3} \oplus_{2} \mathbb{R}$ имеется большой запас троек точек, которые не лежат на одной прямой и для которых верно равенство треугольника. Основные технические детали собраны в следующем предложении.

ПРЕДЛОЖЕНИЕ 2.2. В банаховом пространстве $Y=l_{\infty}^{3} \oplus_{2} \mathbb{R}$ найдется прямая $l \subset l_{\infty}^{3}$ со следущим свойством: для любых положсительных $R$ и $\varepsilon$ в плоскости $\Pi=l \oplus_{2} \mathbb{R}$ существует $q$-паравыпукльй $(0 \leqslant q<1)$ график $A \subset \Pi$ некоторой непрерывной функиии $f: l \rightarrow \mathbb{R}$ такой, что у его замкнутой $\varepsilon$-окрестности $A_{\varepsilon}$ функиия невыпуклости $\alpha_{A_{\varepsilon}}$ равна единице в точке $R$. Более того, $q$ можно считать монотонно возрастающей функиией отношения $R / \varepsilon$ (puc. 1).

В банаховых пространствах с “хорошей” нормой таких геометрически непонятных примеров не существует. 


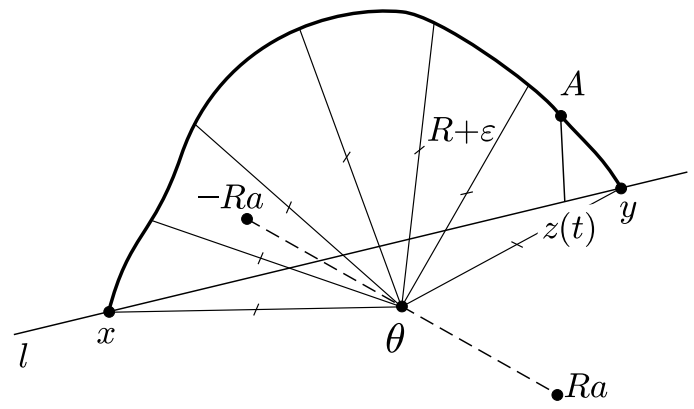

Рис. 1

ТЕОРема 2.3. Для любого равномерно выпуклого банахова пространства $Y$, любой функиии $\alpha(\cdot)<1_{-0}$ и любого $\varepsilon>0$ найдется функиия $\beta(\cdot)<1_{-0}$ такая, что у любого $\alpha$-паравыпуклого множества $P$ его замкнутая $\varepsilon$-окрестность $\bar{D}(P, \varepsilon)$ является $\beta$-паравыпуклым множеством.

Следующее предложение занимает промежуточное между теоремами 2.1 и 2.3 положение. С одной стороны, оно находится в области применимости теоремы 2.3. С другой стороны, оно показывает, что даже на евклидовой плоскости функция $\beta=\beta(\alpha, \varepsilon)$ из теоремы 2.3 может поточечно не стремиться к функции $\alpha$ при $\varepsilon \rightarrow 0$. В то же время, ситуация из теоремы $2.1 \mathrm{c}$ равенством $\beta(\cdot)=1$ в некоторых точках здесь невозможна.

ПРЕДЛОЖЕНИЕ 2.4. Для евклидовой плоскости при любом $0 \leqslant q<1$ найдутся число $p \in(q, 1), q$-паравыпукльй компакт $K$ и последовательность положительных чисел $\varepsilon_{n} \rightarrow 0$ такие, что окрестности $\bar{D}\left(K, \varepsilon_{n}\right)$ не р-паравыпукльь.

\section{3. Доказательство теоремы 2.1.}

ВЫВОД ТЕОРЕМЫ 2.1 ИЗ ПРЕДЛОЖЕНИЯ 2.2. ВоЗьмем ПроИЗвольно числа $\tau_{0}>0$, $\tau \in\left(0, \tau_{0}\right), R>0, \varepsilon>0$ так, чтобы $R / \varepsilon=\tau$. По предложению 2.2 построим $q(\tau)$-паравыпуклое множество $A=A(R, \varepsilon) \subset \Pi=l \oplus_{2} \mathbb{R}$, являющееся графиком некоторой функции $f: l \rightarrow \mathbb{R}$. В силу монотонности $q(\cdot)$ относительно частного $R / \varepsilon$ множество $A$ будет $q\left(\tau_{0}\right)$-паравьпукло, $q\left(\tau_{0}\right)=q_{0}<1$, и, в то же время,

$$
\alpha_{\bar{D}(A, \varepsilon)}(R)=1 \text {. }
$$

Теперь фиксируем последовательность $\varepsilon_{n} \rightarrow 0$ и для каждого $\varepsilon_{n}$ рассмотрим все рациональные числа $R_{n, m} \in\left(0, \varepsilon_{n} \cdot \tau_{0}\right), m \in \mathbb{N}$. Построим, как выше, множества $A_{n, m}=A\left(R_{n, m}, \varepsilon_{n}\right)$, и в евклидовой плоскости П вдоль прямой $l$ разместим изометричные копии (параллельные переносы) этих множеств так, чтобы конщы дуг $A_{n, m}$ лежали бына прямой $l$. При этом разместим это счетное число дуг “страшно далеко" друг от друга, т.е. чтобы расстояния между соседними копиями были бы на порядок больше суммы их диаметров. В промежутках на прямой между соседними копиями проведем отрезки. Получим множество $P \subset \Pi$, являюшееся графиком некоторой непрерывной функции.

Докажем, что это множество $q$-паравьпукло для некоторого $q \in\left(q_{0}, 1\right)$. Используем внутреннюю функцию невьпуклости. Пусть $\Delta$ - произвольньй симплекс с вершинами 
из $P$. Так как $P$ есть график непрерьвной функции, то (см. [3]) можно считать $\Delta$ отрезком. Если его концы из различных копий, то это значит, что длина отрезка $\Delta$ огромна по сравнению с диаметрами этих копий. Поэтому относительное удаление точек отрезка $\Delta$ от множества $P$ оценивается сверху достаточно малым числом. Следовательно, остаются два случая: либо концы отрезка $\Delta$ лежат ровно в одном множестве $A_{n, m}$, либо один конец лежит в $A_{n, m}$, а другой конец лежит на отрезке, выходящем из конца дуги $A_{n, m}$. В первом случае расстояние от точек отрезка $\Delta$ до множества $P$ оценивается в силу $q_{0}$-паравыпуклости дуги $A_{n, m}$. Во втором случае это относительное удаление не превышает $\sqrt{2} / 2$ (см. [3]).

Рассмотрим теперь замкнутую $\varepsilon_{n}$-окрестность $\bar{D}\left(P, \varepsilon_{n}\right)=P_{n}$ множества $P$. По построению для каждого рационального $R_{n, m} \in\left(0, \tau_{n}\right), \tau_{n}=\varepsilon_{n} \tau_{0}$, найдется точка $\theta_{n, m}$, являющаяся серединой некоторого отрезка длины $2 R_{n, m}$ с концами из $P_{n}$ и удаленная от $P_{n}$ на расстояние $R_{n, m}$. Ясно, что минимум расстояний от $\theta_{n, m}$ до прямой $l$ реализуется между концами дуги $A_{n, m}$. Значит, $\alpha_{P_{n}}\left(R_{n, m}\right)=1$ для всех $m \in \mathbb{N}$. По непрерывности функций невьпуклости слева получаем, что функция невьпуклости множества $P_{n}$ тождественно равна единище на интервале $\left(0, \tau_{n}\right)$.

ДОКАЗАТЕЛЬСТВО ПРЕДЛОЖЕНИЯ 2.2. Пусть

$$
\begin{gathered}
a=(1,0,1,0), \quad b=(0,1,1,0), \quad c=(0,0,1,0), \\
x=R a+\varepsilon b=(R, \varepsilon, R+\varepsilon, 0), \quad y=-R a-\varepsilon c=(-R, 0,-R-\varepsilon, 0), \quad l=\operatorname{span}\{x, y\} .
\end{gathered}
$$

Тогда

$$
\|x\|=R+\varepsilon=\|y\|, \quad\|x-y\|=\|(2 R, \varepsilon, 2 R+2 \varepsilon, 0)\|=2 R+2 \varepsilon=\|x\|+\|-y\| .
$$

Для произвольной точки $z(t)=(1-t) x+t y$ отрезка $[x, y]$ ее норма вычисляется явно:

$$
\|z(t)\|=\max \{\varepsilon(1-t),(R+\varepsilon)|1-2 t|\} .
$$

Минимум функции $\|z(t)\|$ достигается в точке $t_{0}=(R+2 \varepsilon) /(2 R+3 \varepsilon)$ и равен

$$
\left\|z_{0}\right\|=\left\|z\left(t_{0}\right)\right\|=\frac{(R+\varepsilon) \varepsilon}{2 R+3 \varepsilon}
$$

Рассмотрим евклидову плоскость $\Pi=l \oplus_{2} \mathbb{R} \subset Y$, и в этой плоскости на отрезке $[x, y]$ рассмотрим график $A$ функции

$$
f(z(t))=\sqrt{(R+\varepsilon)^{2}-\|z(t)\|^{2}}, \quad f:[x, y] \rightarrow \mathbb{R} .
$$

$\Phi$ ункция $f$ унимодальна: она возрастает на $\left[x, z\left(t_{0}\right)\right]$ и убывает на $\left[z\left(t_{0}\right), y\right]$.

Так как концы отрезка $[x, y]$ лежат в $A$, то точки $\pm R a$ лежат в замкнутой $\varepsilon$-окрестности $\bar{D}(A, \varepsilon)=A_{\varepsilon}$ множества $A$. Значит, начало координат $\theta$ лежит в $\operatorname{conv}\left(A_{\varepsilon}\right)$. В то же время, все точки множества $A_{\varepsilon}$ по построению удалены от $\theta$ именно на расстояние $R+\varepsilon$. Следовательно, $\alpha_{A_{\varepsilon}}(R)=1$ и остается проверить паравьпуклость множества $A$, являющегося графиком непрерьвной функции на отрезке.

К сожалению, функция эта не липшицева, а имеется только следуюшая оценка:

$$
\frac{\left|f\left(z\left(t_{1}\right)\right)-f\left(z\left(t_{2}\right)\right)\right|}{\left\|z\left(t_{1}\right)-z\left(t_{2}\right)\right\|}=\frac{\left|\left\|z\left(t_{2}\right)\right\|-\left\|z\left(t_{1}\right)\right\|\right|}{\left\|z\left(t_{1}\right)-z\left(t_{2}\right)\right\|} \cdot \frac{\left\|z\left(t_{2}\right)\right\|+\left\|z\left(t_{1}\right)\right\|}{f\left(z\left(t_{1}\right)\right)+f\left(z\left(t_{2}\right)\right)} \leqslant \frac{2(R+\varepsilon)}{f\left(z\left(t_{1}\right)\right)+f\left(z\left(t_{2}\right)\right)} .
$$

На концах отрезка последняя дробь обращается в бесконечность. Поэтому здесь напрямую не применима техника работ [2], [3]. 
Лемма 3.1. Пусть в предыдущих обозначениях

$$
t_{0}=\frac{R+2 \varepsilon}{2 R+3 \varepsilon}, \quad f_{0}=f\left(z\left(t_{0}\right)\right), \quad p=\frac{f_{0}}{R+\varepsilon}=\sqrt{1-\left(\frac{\varepsilon}{2 R+3 \varepsilon}\right)^{2}},
$$

число $\lambda$ фиксировано на интервале $\left(1, p^{-1}\right)$, а число $\mu$ фиксировано на интервале $\left(0, \sqrt{\lambda^{2}-1} /(p \lambda)\right)$. Тогда график функиии $f$, т.е. множество $A$, является q-паравыпуклым множеством, где

$$
q=\max \left\{p \lambda, \sin \left(\operatorname{arctg}\left(L^{\prime}\right)\right)\right\}, \quad L^{\prime}=\operatorname{tg} \frac{\operatorname{arctg}(L)+\pi / 2}{2}, \quad L=\frac{1}{p \mu}
$$

(cM. puc. 2).

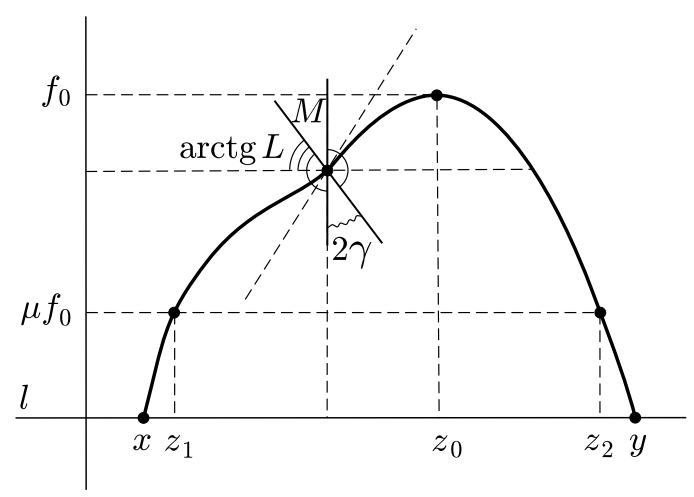

Рис. 2

ДокАЗАТЕЛЬСтво. Проведем горизонталь на высоте $\mu f_{0}$. Имеются ровно две точки $z_{1}<z_{2}$ на отрезке $[x, y]$, в которых функция $f$ принимает значение $\mu f_{0}$. Найдем разность $\left\|z_{2}-z_{1}\right\|$. Имеем

$$
\begin{gathered}
f\left(z_{i}\right)=(R+\varepsilon) \sqrt{1-\left(1-2 t_{i}\right)^{2}}=\mu f_{0}=\mu(R+\varepsilon) p \\
\left(1-2 t_{i}\right)^{2}=1-\mu^{2} p^{2}, \quad\left|t_{2}-t_{1}\right|=\sqrt{1-\mu^{2} p^{2}} .
\end{gathered}
$$

Значит,

$$
\begin{aligned}
\left\|z_{2}-z_{1}\right\| & =\left\|\left(1-t_{2}\right) x+t_{2} y-\left(1-t_{1}\right) x-t_{1} y\right\|=\left|t_{2}-t_{1}\right| \cdot\|x-y\| \\
& =\left|t_{2}-t_{1}\right| \cdot 2(R+\varepsilon)=2 \sqrt{1-\mu^{2} p^{2}}(R+\varepsilon) .
\end{aligned}
$$

Для оценки паравыпуклости графика любой непрерывной функции достаточно рассматривать только отрезки с концами на графике [3]. Более того, достаточно получить верхнюю оценку для удаления только середин этих отрезков от графика функции. Действительно, тогда для точек отрезка, близких к середине, оценка будет почти такой же, а точки отрезка, заметно отличающиеся от середины, будут достаточно близки к концам 
отрезка, лежащим на графике. Используем также следующее простое геометрическое наблюдение [3]. Если конщы отрезка длины $2 r$ лежат на графике липшицевой с константой $K$ функции, то середина этого отрезка удалена от графика функции не более, чем на $\sin (\operatorname{arctg}(K)) \cdot r$.

Вернемся к нашей функции $f:[x, y] \rightarrow \mathbb{R}$. Возьмем любой отрезок с концами на графике этой функции. Если спроектировать такой отрезок на "горизонтальную" ось $\operatorname{span}\{x, y\}$, то возможны ровно три ситуации:

а) левьй конец попал в $\left[x, z_{1}\right]$, а правьй - в $\left[z_{2}, y\right]$;

б) правьй конец попал в $\left[x, z_{2}\right]$;

в) левьй конец попал в $\left[z_{1}, y\right]$.

Случай а). Длина отрезка здесь не меньше $\left\|z_{2}-z_{1}\right\|$, а расстояние от точек отрезка до графика функции не больше $f_{0}$. Значит, относительное удаление середины этого отрезка от графика функции $f$ данном случае оценивается сверху отношением

$$
\frac{2 f_{0}}{\left\|z_{2}-z_{1}\right\|}=\frac{p}{\sqrt{1-\mu^{2} p^{2}}}<\lambda p
$$

где последнее неравенство обеспечено выбором $\mu \in\left(0, \sqrt{\lambda^{2}-1} /(p \lambda)\right)$.

Случай б). Заметим сначала, что для ограничения на отрезок $\left[z_{1}, z_{2}\right]$ липшицевость функции $f$ уже оценена выше: для любых $t_{1}^{\prime}$ и $t_{2}^{\prime}$ из отрезка $\left[t_{1}, t_{1}\right]$ верно неравенство

$$
\frac{\left|f\left(z\left(t_{1}^{\prime}\right)\right)-f\left(z\left(t_{2}^{\prime}\right)\right)\right|}{\left\|z\left(t_{1}^{\prime}\right)-z\left(t_{2}^{\prime}\right)\right\|} \leqslant \frac{2(R+\varepsilon)}{f\left(z\left(t_{1}^{\prime}\right)\right)+f\left(z\left(t_{2}^{\prime}\right)\right)} \leqslant \frac{2(R+\varepsilon)}{f\left(z_{1}\right)+f\left(z_{2}\right)}=\frac{R+\varepsilon}{\mu f_{0}}=\frac{1}{\mu p}=L .
$$

Для любой точки $M$ графика с горизонтальной проекцией, лежащей на $\left[x, z_{2}\right]$, построим сначала следующий угол с вершиной в этой точке, смотрящий влево. Одна сторона - это вертикаль, направленная вниз. Другая сторона - это луч с отрицательньп тангенсом угла наклона, по величине равным $\operatorname{arctg}(L)$. Угол этот, разумеется, меньше развернутого. Утверждается, что весь график функции $f$ левее точки $M$ лежит в построенном угле, величина которого равна

$$
\frac{\pi}{2}+\operatorname{arctg}(L)=2(\operatorname{arctg}(L)+\gamma), \quad \gamma=\frac{\pi / 2-\operatorname{arctg}(L)}{2}
$$

Для точек графика, у которых горизонтальная проекция больше $z_{1}$, это верно согласно случаю а). Для точек графика на отрезке $\left[x, z_{1}\right]$ это верно, так как на этом отрезке функция $f$ возрастает и левее любой своей точки график лежит в третьей координатной четверти.

Правее точки $M$ график функции $f$ (до точки $z_{2}$ ) лежит в угле величины $2 \operatorname{arctg}(L)$ с вершиной в $M$ и горизонтальной биссектриссой. Значит, он тем более попадет в угол, смотрящий вправо, у которого одна сторона - вертикаль, направленная вверх, а другая сторона есть продолжение наклонной стороны построенного выше, смотрящего влево угла. Другими словами, построены два симметричных относительно точки $M$ угла, каждьй из которых равен $2(\operatorname{arctg}(L)+\gamma)$ и в объединении которых содержится график функции $f$ на отрезке $\left[x, z_{2}\right]$. 
В итоге, если повернуть систему координат против часовой стрелки на угол $\gamma$, то в новой системе координат нужньй нам участок графика функции $f$ будет графиком липшицевой функции с константой

$$
L^{\prime}=\operatorname{tg}(\operatorname{arctg}(L)+\gamma)=\operatorname{tg} \frac{\operatorname{arctg}(L)+\pi / 2}{2}
$$

Значит, относительное удаление середины любого отрезка с концами на графике функции $f$ на отрезке $\left[x, z_{2}\right]$ оценивается сверху числом $\sin \left(\operatorname{arctg}\left(L^{\prime}\right)\right)$.

Случай в) рассматривается аналогично.

Лемма доказана.

Вернемся к доказательству предложения 2.2. Остается явно выразить индекс паравыпуклости $q$ множества $A$ через $R, \varepsilon, \tau=R / \varepsilon$. Итак, по определению

$$
p=\sqrt{1-\left(\frac{\varepsilon}{2 R+3 \varepsilon}\right)^{2}}=\sqrt{1-\left(\frac{1}{2 \tau+3}\right)^{2}} .
$$

Значит, $p=p(\tau)$ монотонно возрастает от $p(0)=2 \sqrt{2} / 3$ до $p(\infty)=1$. Далее, в соответствии с лемой 3.1 функцию $\lambda=\lambda(\tau)$ можно считать равной $(p+1) /(2 p)$, а функцию $\mu=\mu(\tau)$ можно считать равной $\sqrt{\lambda^{2}-1} /(2 p \lambda)$. Тогда и $\lambda$, и $\mu$ монотонно убывают, а

$$
L=\frac{1}{\mu p}, \quad L^{\prime}=\operatorname{tg} \frac{\operatorname{arctg}(L)+\pi / 2}{2} \quad \text { и } \quad \lambda p=\frac{p+1}{2}
$$

монотонно возрастают. Следовательно, и верхнюю оценку

$$
q(\tau)=q=\max \left\{\lambda p, \sin \left(\operatorname{arctg}\left(L^{\prime}\right)\right)\right\}
$$

функции невыпуклости множества $A$ можно считать монотонно возрастающей относительно переменной $\tau=R / \varepsilon$. Предложение 2.2 и теорема 2.1 доказаны.

4. Доказательства теоремы 2.3 и предложения 2.4. Для дальнейшего потребуется следующая лемма, доказанная в [9] для случая гильбертова пространства. Грубо говоря, она утверждает, что “высоты” треугольника малы, если одна из его сторон почти равна сумме двух других сторон. Напомним, что равномерная вьпуклость банахова пространства $Y$ означает положительность модуля выпуклости единичной сферы этого пространства:

$$
0<\delta(\varepsilon)=\inf \left\{1-\left\|\frac{x+y}{2}\right\|:\|x\|=\|y\|=1,\|x-y\| \geqslant \varepsilon\right\}, \quad \varepsilon \in(0,2]
$$




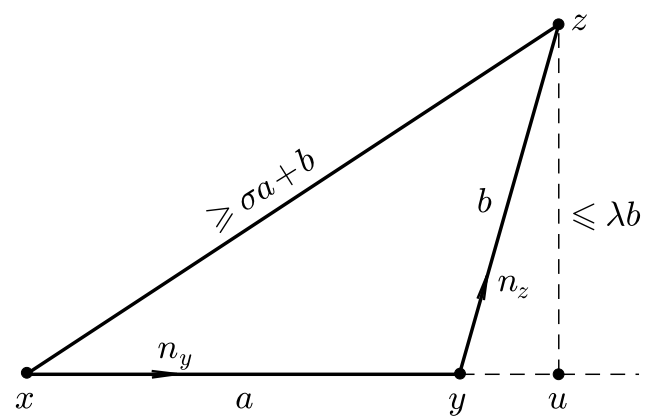

Рис. 3

Лемма 4.1. Пусть $(Y,\|\cdot\|)$ - равномерно выпуклое банахово пространство, и пусть $\lambda>0$. Тогда существует непрерывная функиия $\sigma_{\lambda}:(0, \infty) \times(0, \infty) \rightarrow(0,1)$ такая, что из $\|x-y\|=a,\|y-z\|=b,\|x-z\| \geqslant \sigma_{\lambda}(a, b) \cdot a+b$ следует неравенство $\operatorname{dist}(z, \operatorname{span}\{x, y\}) \leqslant \lambda b$ (c.M. puc. 3$)$.

ДокАЗАТЕЛЬСТвО. Пусть $n_{y}$ и $n_{z}$ - единичные векторы, сонаправленные с $y-x$ и с $z-y$ соответственно. Тогда

$$
y=x+a n_{y}, \quad z=y+b n_{z}=x+a n_{y}+b n_{z} .
$$

На прямой, проходящей через $x$ и $y$, возьмем точку $u=t x+(1-t) y \mathrm{c} t=-b / a$ и найдем расстояние между $z$ и $u$. Имеем

$$
\begin{aligned}
\|z-u\| & =\|t z+(1-t) z-t x-(1-t) y\|=\|t(z-x)+(1-t)(z-y)\| \\
& =\left\|t\left(a n_{y}+b n_{z}\right)+(1-t) b n_{z}\right\|=b\left\|n_{y}-n_{z}\right\| .
\end{aligned}
$$

Значит, $\operatorname{dist}(z, \operatorname{span}\{x, y\}) \leqslant b\left\|n_{y}-n_{z}\right\|$. Остается добиться того, чтобы $\left\|n_{y}-n_{z}\right\| \leqslant \lambda$ или $\delta\left(\left\|n_{y}-n_{z}\right\|\right) \leqslant \delta(\lambda)$, где функция $\delta(\cdot)$ есть модуль вьпуклости единичной сферы пространства $Y$.

Рассмотрим сначала случай $a \geqslant b$. Решающий технический момент (добавление и вычитание $b n_{y}$ ) указал авторам В. М. Кадец, которому мы выражаем признательность. Имеем

$$
\begin{aligned}
\sigma_{\lambda}(a, b) a+b & \leqslant\|z-x\|=\left\|a n_{y}+b n_{z}+b n_{y}-b n_{y}\right\| \leqslant(a-b)+2\left\|\frac{n_{y}+n_{z}}{2}\right\| \\
& \leqslant(a-b)+2 b\left(1-\delta\left(\left\|n_{y}-n_{z}\right\|\right)\right)=a+b-2 b \delta\left(\left\|n_{y}-n_{z}\right\|\right) .
\end{aligned}
$$

Значит,

$$
\delta\left(\left\|n_{y}-n_{z}\right\|\right) \leqslant \frac{a}{2 b}\left(1-\sigma_{\lambda}(a, b)\right) .
$$

Поэтому для того чтобы выполнялось неравенство $\delta\left(\left\|n_{y}-n_{z}\right\|\right) \leqslant \delta(\lambda)$, достаточно добиться того, что

$$
\frac{a}{2 b}\left(1-\sigma_{\lambda}(a, b)\right) \leqslant \delta(\lambda) .
$$


Другими словами, формула

$$
\sigma_{\lambda}(a, b)=\max \left\{0,1-\frac{2 b}{a} \delta(\lambda)\right\}
$$

дает нужньй ответ.

Покажем теперь, что для $a<b$ можно положить $\sigma(a, b)=\sigma(a, a)$. Рассмотрим треугольник с вершинами $z, x, y+a n_{z}$. В нем сторона $\left[x, y+a n_{z}\right]$ по длине не меньше разности двух других сторон, и поэтому

$$
\left\|y+a n_{z}-x\right\| \geqslant\|z-x\|-(b-a)\left\|n_{z}\right\| \geqslant \sigma(a, b) a+b-b+a=\sigma(a, a) a+a .
$$

Значит, к треугольнику с вершинами $x, y, y+a n_{z}$ применим предыдущий случай, и поэтому расстояние от точки $y+a n_{z}$ до прямой $\operatorname{span}\{x, y\}$ не превьшает числа $\lambda \cdot a$. Рассматривая гомотетию с коэффициентом $b / a$ и центром $y$, получаем, что расстояние от точки $z$ до прямой $\operatorname{span}\{x, y\}$ не превышает числа $\lambda \cdot b$.

Отметим, что в силу непрерьвности модуля вьпуклости построенная функция $\sigma$ будет непрерывной и по переменной $\lambda$.

Равномерная вьпуклость банахова пространства гарантирует сохранение многих геометрических свойств, которые имеют место в гильбертовом пространстве. Например, расстояние от фиксированной точки до выпуклого замкнутого множества единственным образом реализуется в некоторой точке этого множества. Или же, ограниченное подмножество равномерно выпуклого банахова пространства имеет единственный чебьшевский центр [10]. Нам потребуется еще одно свойство такого рода: если выпуклая оболочка $(n+1)$-элементного множества имеет размерность $n$ и если чебьшевский центр этой оболочки лежит внутри нее, то все расстояния от чебышевского центра до точек этого множества равны чебьшевскому радиусу этого множества.

При доказательстве теоремы 2.3 мы, по-существу, будем оценивать не саму функцию невыпуклости множества, но его внутреннюю центральную функцию невьпуклости. Другими словами, мы будем брать конечные подмножества данного множества, рассматривать их чебьшевские центры и оценивать удаление этих центров от самого множества некоторой подходящей числовой функцией от чебышевского радиуса этого конечного множества.

ДОКАЗАТЕЛЬСТво тЕОРЕмЫ 2.3. Пусть $P$ - множество, функция невыпуклости которого $\alpha_{P}$ мажорируется на луче $(0, \infty)$ функцией $\alpha<1_{-0}$. Оценим сверху функцию невыпуклости множества $\bar{D}(P, \varepsilon)=Q$ на этом же луче. Фиксируем произвольньй открытый шар $D_{r}=D$ радиуса $r$, пересекаюший множество $Q$.

Пусть $K=\left\{y_{1}, \ldots, y_{n}\right\} \subset D \cap Q$, и пусть $y \in \operatorname{conv}\left\{y_{1}, \ldots, y_{n}\right\}$. Нам следует оценить сверху расстояние $\operatorname{dist}(y, Q)$. Для этого фиксируем точки $z_{i} \in P, \varepsilon$-близкие к соответствуюшим точкам $y_{i}$. Если какое-то из расстояний $\left\|y_{i}-z_{i}\right\|$ меньше $\varepsilon$, то на полуинтервале $\left[y_{i}, z_{i}\right)$ возьмем точку $u_{i}$ так, чтобы $\left\|y_{i}-u_{i}\right\|=\varepsilon$.

Рассмотрим ситуацию, когда точка $y$ есть чебьшевский центр (единственньй в силу равномерной вьпуклости единичной сферы пространства $Y$ ) многогранника $\operatorname{conv}\left\{y_{1}, \ldots\right.$ $\left.\ldots, y_{n}\right\}$. Если размерность многогранника $\operatorname{conv}\left\{y_{1}, \ldots, y_{n}\right\}$ меньше $n-1$ или если $y$ 
лежит на гранище этого многогранника, то можно перейти к меньшему числу точек и проводить рассуждение по индукции. Поэтому достаточно рассматривать случай, когда все расстояния $\left\|y-y_{i}\right\|$ равны одному и тому же числу - чебьшевскому радиусу $r$ этого многогранника.

Итак, у нас фиксированы функция $\alpha<1_{-0}$ и положительные числа $r$ и $\varepsilon$. Положим $\lambda=1-\alpha(r+\varepsilon) \in(0,1]$ и рассмотрим функцию $\sigma_{\lambda}(\cdot, \cdot)$, построенную в лемме 4.1. Возможны в точности следующие три случая:

а) $\sigma(r, \varepsilon) r+\varepsilon<r$ и для некоторого $i$ верно неравенство $\left\|y-u_{i}\right\|<\sigma(r, \varepsilon) r+\varepsilon$;

б) $r \leqslant \sigma(r, \varepsilon) r+\varepsilon$ и для некоторого $i$ верно неравенство $\left\|y-u_{i}\right\|<\sigma(r, \varepsilon) r+\varepsilon$;

в) для всех точек $u_{i}$ верно неравенство $\left\|y-u_{i}\right\| \geqslant \sigma(r, \varepsilon) r+\varepsilon$.

В случае а) в треугольнике $\Delta y y_{i} u_{i}$ одна из сторон с вершиной $y$ равна $r$, а другая меньше. Тогда в силу равномерной вьпуклости получаем, что $\operatorname{dist}(y, P) \leqslant\left\|y-z_{i}\right\|<r$ и, значит, $\operatorname{dist}(y, Q)$ равно нулю при $r \leqslant \varepsilon$ и меньше $r-\varepsilon$ при $r>\varepsilon$. В итоге в этом случае получаем, что

$$
\frac{\operatorname{dist}(y, Q)}{r} \leqslant \max \left\{0,1-\frac{\varepsilon}{r}\right\} .
$$

В случае б) рассмотрение того же треугольника дает оценку

$$
\operatorname{dist}(y, P) \leqslant\left\|y-z_{i}\right\|<\sigma(r, \varepsilon) r+\varepsilon,
$$

откуда следует

$$
\frac{\operatorname{dist}(y, Q)}{r}<\sigma(r, \varepsilon) .
$$

В случае в) к каждому из треугольников $\Delta y_{i} u_{i}$ применима лемма $4.1 \mathrm{c} \lambda=$ $1-\alpha(r+\varepsilon) \in(0,1]$. Тогда точки $u_{i}$ и, тем более, точки $z_{i}$ будут $(\lambda \varepsilon)$-близки к прямым, проходящим через точки $y$ и $y_{i}$, и при этом точки $y_{i}^{\prime}$, реализующие такое удаление, будут конщами отрезков $\left[y, y_{i}^{\prime}\right]$, содержащих точки $y_{i}$. Значит, точка $y$ лежит в вьпуклой оболочке conv $\left\{y_{1}^{\prime}, y_{2}^{\prime}, \ldots, y_{n}^{\prime}\right\}$ точек, каждая из которых $(\lambda \varepsilon)$-близка ко множеству $P$.

Следовательно, точка $y$ будет $(\lambda \varepsilon)$-близка к некоторой точке $z$ многогранника $\operatorname{conv}\left\{z_{1}, \ldots, z_{n}\right\}$. Но для таких точек по условию имеется следующая оценка для удаления от множества $P$ :

$$
\operatorname{dist}(z, P) \leqslant \alpha_{P}(r+\varepsilon) \cdot(r+\varepsilon)<\alpha(r+\varepsilon) \cdot(r+\varepsilon) .
$$

Значит, $\operatorname{dist}(y, P)<\lambda \varepsilon+\alpha(r+\varepsilon) \cdot(r+\varepsilon)=(1-\alpha)(r+\varepsilon) \cdot \varepsilon+\alpha(r+\varepsilon) \cdot(r+\varepsilon)=\varepsilon+\alpha(r+\varepsilon) \cdot r$ в силу выбора параметра $\lambda$. Следовательно, $\operatorname{dist}(y, Q) / r<\alpha(r+\varepsilon)$. В итоге, имеем

$$
\frac{\operatorname{dist}(y, Q)}{r}<\max \left\{\alpha(r+\varepsilon), \sigma_{\lambda}(r, \varepsilon), 1-\frac{\varepsilon}{r}, 0\right\} .
$$

Правая часть последнего неравенства и дает ответ для искомой верхней оценки $\beta(\cdot)<1_{-0}$ иентральной функции невыпуклости множества $Q$. Тогда по лемме 14 из [9] и для обычной функции невьпуклости этого множества имеется некоторая геометрически суммируемая мажоранта, зависящая только от найденной мажоранты $\beta(\cdot)$ и от модуля равномерной вьпуклости пространства $Y$. Теорема 2.3 доказана. 
ДоКАЗАТЕЛЬСТВо ПРЕДЛОЖЕНИЯ 2.4. Для фиксированного $0 \leqslant q<1$ определим число $p \in(q, 1)$ как решение следующего уравнения:

$$
\frac{\sqrt{1+q^{2}}-1}{p-q}=2 \cdot \frac{p-q / \sqrt{1+q^{2}}}{1-p^{2}}
$$

Обе части этого уравнения непрерьвны относительно переменной $p \in(q, 1)$. При этом при $p=1$ левая часть конечна, а правая бесконечна, а при $p=q$ - наоборот. Значит, решения этого уравнения в интервале $(q, 1)$ действительно существуют.

Для произвольного $\varepsilon>0$ в евклидовой плоскости $\mathbb{R}^{2}$ построим точки $C_{ \pm}=( \pm R, 0)$, где $R$ равняется произведению числа $\varepsilon$ и левой (= правой) части вьшеуказанного уравнения. Точки $A_{ \pm}$определим как сдвиги точек $C_{ \pm}$на $\pm \varepsilon q / \sqrt{1+q^{2}}$ по оси $O X$ и поднятие на $1 / \sqrt{1+q^{2}}$ по оси $O Y$, а точки $B_{ \pm}$определим как поднятия точек $A_{ \pm}$по оси $O Y$ на $q \cdot x\left(A_{+}\right)$.

Множество $K_{\varepsilon}$ определим как ломаную $A_{-} B_{-} B_{+} A_{+}$. По построению это множество $q$-паравыпукло. Кроме того, точки $C_{ \pm} \varepsilon$-близки к точкам $A_{ \pm}$и, значит, начало координат $\theta$ лежит в $\operatorname{conv}\left(\bar{D}\left(K_{\varepsilon}, \varepsilon\right)\right)$. Ясно, что минимумы расстояний между $\theta$ и точками множества $K_{\varepsilon}$ достигаются в концах $A_{ \pm}$множества $K_{\varepsilon}$ и в точке $B=\left(B_{-}+B_{+}\right) / 2$. Но числа $R>0$ и $p \in(q, 1)$ подобраны именно так, что

$$
\operatorname{dist}\left(\theta, A_{ \pm}\right)=\operatorname{dist}(\theta, B)=p R+\varepsilon
$$

Значит, $\operatorname{dist}\left(\theta, \bar{D}\left(K_{\varepsilon}, \varepsilon\right)\right)=p R$ и множество $\bar{D}\left(K_{\varepsilon}, \varepsilon\right)$ не $p$-паравыпукло: значение его (замкнутой) функции невьпуклости в точке $R$ в точности равно $p$.

Отметим, что для гомотетичной с коэффициентом $\lambda>0$ копии множества $K_{\varepsilon}$ аналогичньй эффект будет наблюдаться для значения функции невьпуклости его замкнутой $(\lambda \varepsilon)$-окрестности в точке $\lambda R$. Остается взять сходящийся ряд из положительных чисел $\lambda_{n}$, на фиксированной прямой разместить $\lambda_{n}$-гомотетичные копии множества $K_{\varepsilon}$ на подходящих расстояниях, добавить отрезки между ними и еще одну предельную точку. Полученньй компакт $K$ будет $q$-паравьпукл, но для каждого $n \in \mathbb{N}$ значение функция невьпуклости замкнутой $\left(\lambda_{n} \varepsilon\right)$-окрестности этого множества будет равно $p>q$ в точке $\lambda_{n} R$. Предложение 2.4 доказано.

5. Заключение. Для полунепрерьвных сверху многозначных отображений редукция их исследования к случаю однозначных отображений, как правило, производится с помощью (графических) аппроксимаций, а не селекций. Однозначное отображение $f: X \rightarrow Y$ метрических пространств называется $\varepsilon$ - аппроксим аиией многозначного отображения $F: X \rightarrow 2^{Y}$, если график отображения $f$ лежит в $(\varepsilon \times \varepsilon)$-окрестностиграфика отображения $F$. Аппроксимируемость (т.е. наличие $\varepsilon$-аппроксимаций для всех $\varepsilon>0$ ) замкнутозначного полунепрерьвного сверху отображения компактного абсолютного экстензора (= $A E) X$ в себя гарантирует существование неподвижных точек этого отображения [4]-[7]. В случае $n$-мерного (не обязательно компактного) $X$ апшроксимируемость полунепрерьвного сверху компактнозначного отображения $F: X \rightarrow Y$ является следствием чисто топологических, так назьваемых, $U V^{n}$-свойств значений $F(x)$ : для всякой окрестности $U$ множества $F(x)$ должна существовать его меньшая окрестность $V$, 
для которой тождественное включение $V \hookrightarrow U$ гомотопически тривиально в размерности $n$. В самом общем виде это недавно доказали Щепин и Бродский [11], используя идеи работ [12], [13]. Для бесконечномерного компактного абсолютного окрестностного экстензора (=ANE)-пространства $X$ апшроксимируемость отображения $F: X \rightarrow X$ гарантируется $U V(\infty)$-свойствами значений этого отображения; этот результат принадлежит Гранасу, Гурневичу, Крижевскому [14]. Обобщение для случая, когда $X$ локально конечномерно полиэдрален, получено Крижевским [15]. Для произвольной бесконечномерной области определения $X$ проблема апшоксимируемости не имеет чисто топологического решения даже для компактны $X$. Дело в том, что аппроксимируемые клеточноподобные сюръекции компактов не повышают лебегову размерность [16], а для просто клеточноподобных сюръекций такое повышение возможно [17]. В то же время, добавление условия выпуклозначости отображения $F$ сразу дает решение этой проблемы, даже без условия его компактнозначности - это классическая аппроксимационная теорема фон Неймана-Челлины [6], [7].

Поэтому для нахождения невыпуклозначного аналога аппроксимационной теоремы фон Неймана-Челлины для бесконечномерной области определения естественно и возник вопрос о корреляции между характером невьпуклости множества и его $\varepsilon$-окрестностей. Теорема 2.3 показывает, что окрестности паравьпуклых подмножеств равномерно выпуклых банаховых пространств топологически тривиальны. В частности, такие паравьпуклые множества являются $U V(\infty)$-подмножествами объемлющего пространства. Как следствие получаем, что всякое полунепрерьвное сверху $\alpha$-паравьпуклозначное отображение метрического пространства в равномерно выпуклое банахово пространство является аппроксимируемьм; $\alpha<1_{-0}$. Мы полагаем, что этот аппроксимационньй факт верен для любого нормированного пространства.

В заключение, несколько слов о паравыпуклости с наперед заданной точностью (см. определение 1.1(в)). Дело в том, что паравьпуклость не является устойчивым свойством относительно метрики Хаусдорфа в экспоненте банахова пространства. Малые (относительно матрики Хаусдорфа) шевеления паравьпуклого множества могут быть уже не только не паравьпуклыми, но и быть топологически нетривиальньми. В противоположность этому паравыпуклость с фиксированной точностью уже обладает такой устойчивостью. Неравенство $\operatorname{Hausd}(P, Q)<\lambda$ ниже означает, что множества $P$ и $Q$ лежат в $\lambda$-окрестностях друг друга.

ПРЕДЛОЖЕНИЕ 5.1. ДЛя Кажсдого нормированного пространства $Y$ и любъх чисел $q \in[0,1), \delta>0, p \in(q, 1)$ существует число $\lambda \in(0, \delta)$ такое, что для каждого q-паравыпуклого с точностью $\delta$ подмножества $P \subset Y$ из неравенства $\operatorname{Hausd}(P, Q)<\lambda$ следует, что множество $Q$ является $p$-паравыпукльм с той же точностью $\delta$.

Наконец отметим, что аналог теоремы 2.3 верен и для паравыпуклости с наперед заданной точностью.

Теорема 5.2. Для любого равномерно выпуклого банахова пространства $Y$, любой функиии $\alpha(\cdot)<1_{-0}$ и любых $\varepsilon>0, \delta>0$ найдется функция $\beta(\cdot)<1_{-0}$ такая, что у любого $\alpha$-паравыпуклого с точностью $\delta$ множсества $P$ его замкнутая $\varepsilon$-окрестность $\bar{D}(P, \varepsilon)$ является $\beta$-паравыпукльм с точностью $\delta$ множеством. 
Доказательство теоремы 5.2 повторяет доказательство теоремы 2.3 с небольшой поправкой: всюду следует рассматривать только те конечные подмножества, у которых чебьшевский радиус больше данной точности $\delta$.

Авторы признательны рецензенту работы за сделанные им замечания и предложения.

\section{СПИСОК ЦИТИРОВАННОЙ ЛИТЕРАТУРЫ}

[1] Michael E. Paraconvex sets // Scand. Math. 1959. V. 7. P. 372-376.

[2] Семенов П. В. Функционально паравыпуклые множества // Матем. заметки. 1993. Т. 54. №6. C. $74-81$.

[3] Repovš D., Semenov P. V. On functions of nonconvexity for graphs of continuous functions // J. Math. Anal. Appl. 1995. V. 196. P. 1021-1029.

[4] Борисович Ю. Г., Гельман Б. Д., Мышкис А. Д., Обуховский В. В. Многозначные отображения // Итоги науки и техники. Матем. анализ. 1982. Т. 19. С. 127-231.

[5] Борисович Ю.Г., Гельман Б. Д., Мышкис А. Д., Обуховский В.В. О новых результатах в теории многозначных отображений. I // Итоги науки и техники. Матем. анализ. 1987. T. 25. C. $123-197$.

[6] Aubin J.-P., Cellina A. Differential Inclusions. Set-valued Maps and Viability Theory. Grundlehren Math. Wiss. V. 264. Berlin: Springer Verlag, 1984.

[7] Dugundji J., Granas A. Fixed Point Theory. Vol. I. Monogr. Math. V. 61. Warzsawa: PWN, 1982.

[8] Repovš D., Semenov P. V. Continuous Selections of Multivalued Mappings. Mathematics and its Applications. V. 455. Dordrecht: Kluwer, 1998.

[9] Семенов П. В. Теоремы о неподвижной точке при контролируемом отказе от выпуклости значений многозначного отображения // Матем. сб. 1998. Т. 189. № 3. С. 141-160.

[10] Гаркави А. Л. Теория аппроксимаций в нормированных линейных пространствах // Итоги науки и техники. Математический анализ. Т. 8. М.: ВИНИТИ, 1967. С. 75-132.

[11] Шепин Е. В., Бродский Н. Б. Селекции фильтрованных многозначных отображений // Тр. МИАН. 1996. Т. 212. С. 220-240.

[12] Чернавский А. В., Компанийц В. П. Эквивалентность двух классов отображений сферы // Докл. АН СССР. 1969. Т. 169. №6. С. 1266-1268.

[13] Lacher R. Cell-like spaces // Proc. Amer. Math. Soc. 1969. V. 20. P. 598-602.

[14] Gorniewicz L., Granas A., Kryszewsky W. On the homotopy method in the fixed point index theory of multivalued mappings of compact absolute neighborhood retracts // J. Math. Anal. Appl. 1991. V. 161. P. 457-473.

[15] Kryszewsky W. Graph-approximation of set-valued maps on noncompact domains // Topology Appl. 1998. V. 83. P. 1-21.

[16] Дранишников А.Н., Шепин Е. В. Клеточноподобные отображения. Проблема повышения размерности // УМН. 1986. Т. 41. №6. С. 49-90.

[17] Dranishnikov A. N. $K$-theory of Eilenberg-Maclane spaces and cell-like mapping problem // Trans. Amer. Math. Soc. 1993. V. 335. P. 91-103.

(Д. Реповш) University of Ljubljana, Slovenia

Поступило

(П. В. Семёнов) Московский государственный педагогический университет

18.03 .1999

E-mail: dusan.repovs@fmf.uni-lj.si, pavels@orc.ru

Исправленный вариант

17.11 .2000 